\title{
Rhabdomyosarcoma arising within the biliary tract mimicking a choledochal cyst: A case report
}

\author{
Yesenia Rojas ${ }^{1}$, Bethany J. Slater ${ }^{1}$, M. John Hicks ${ }^{2}$, M. Fatih $\mathrm{Okcu}^{3}$, Prakash Masand ${ }^{4}$, Mark V. Mazziotti ${ }^{* 1}$ \\ ${ }^{1}$ Division of Pediatric Surgery, Michael E. DeBakey Department of Surgery, Baylor College of Medicine, Houston, TX, USA \\ ${ }^{2}$ Department of Pathology and Immunology, Baylor College of Medicine, Houston, TX, USA \\ ${ }^{3}$ Division of Hematology-Oncology, Department of Pediatrics, Baylor College of Medicine, Houston, TX, USA \\ ${ }^{4}$ Department of Radiology, Baylor College of Medicine, Houston, TX, USA
}

Received: November 22, 2015

DOI: $10.5430 /$ crcp.v3n1p65
Accepted: January 25, $2016 \quad$ Online Published: February 16, 2016

URL: http://dx.doi.org/10.5430/crcp.v3n1p65

\begin{abstract}
Rhabdomyosarcoma (RMS) involving the biliary tree and choledochal cysts presents with jaundice and abdominal pain. Imaging studies including ultrasound (US), computerized tomography (CT) scan, and magnetic resonance cholangiopancreatography (MRCP) are useful in the diagnosis and evaluation of biliary tree anatomy. We present a patient with biliary RMS initially thought to be a choledochal cyst with intraluminal cystic debris (biliary sludge) that was diagnosed by frozen section examination during cyst resection and subsequently treated with post-operative chemotherapy and radiation.
\end{abstract}

Key Words: Rhabdomyosarcoma, Biliary tract, Choledochal cysts, Children

\section{INTRODUCTION}

Rhabdomyosarcoma (RMS) is a soft tissue sarcoma of childhood, with an incidence of 4.3 per million per year in children less than 20 years of age. ${ }^{[1]}$ Biliary RMS is rare, accounting for less than $1 \%$ of all RMS in childhood, and often results in a delay in diagnosis because the majority of patients are initially diagnosed with choledochal cysts or infectious hepatitis. ${ }^{[2]}$ Given the rarity of this disease, the majority of the pediatric literature consists of isolated case reports. ${ }^{[3-7]}$ We present a case of a child with biliary RMS initially diagnosed as a choledochal cyst on radiologic imaging.

\section{CASE REPORT}

A 4-year-old previously healthy male presented with a several day history of abdominal pain, decreased oral intake, and pale stools. On physical examination, he was afebrile, had icteric sclera, and mild right upper quadrant abdominal tenderness. Laboratory values were significant for an elevated conjugated bilirubin of $2.3 \mathrm{mg} / \mathrm{dl}$ and mildly elevated transaminases. Radiologic workup included an abdominal ultrasound (US) demonstrating a dilated extrahepatic bile duct with echogenic contents and associated intrahepatic bile duct dilation. Duplex doppler evaluation was not useful in determining presence of internal flow within the echogenic area, due to patient motion. A subsequent magnetic resonance cholangiopancreatography (MRCP) showed severe diffuse dilatation of the entire extra-hepatic biliary system with extension of the dilatation centrally involving the common hepatic, right and left hepatic ducts consistent with a choledochal cyst (see Figure 1). The dilated extrahepatic bile duct showed iso to hypointense (signal compared to liver parenchyma) material within, and the post-contrast imag-

\footnotetext{
*Correspondence: Mark V. Mazziotti; Email: mvmazzio@texaschildrens.org; Address: Division of Pediatric Surgery, 6701 Fannin, Suite CC.1210, Houston, TX 77030-2399, USA.
} 
ing was inconclusive for presence of enhancement. The low signal contents were thought to represent thick sludgelike material within a choledochal cyst. The patient was discharged from the hospital with plans for an elective outpatient resection of the presumed type IV choledochal cyst.
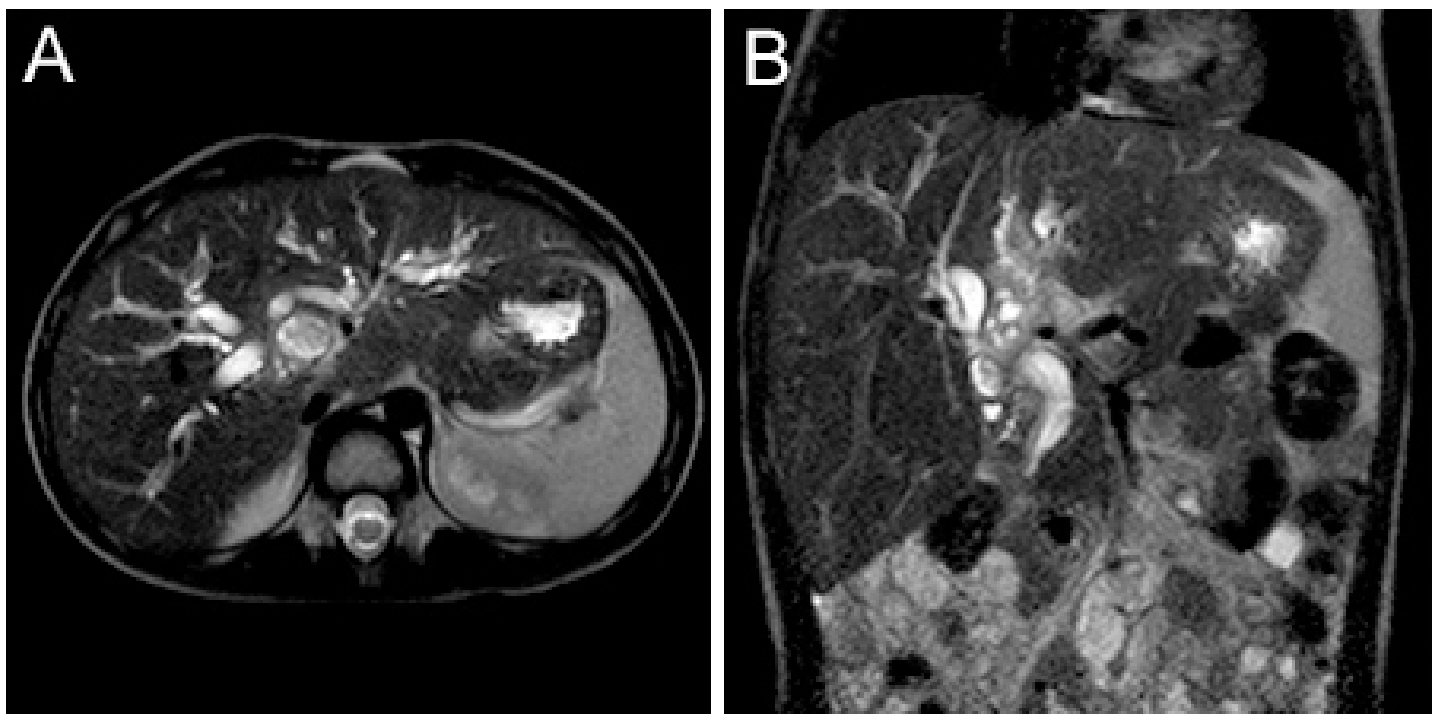

Figure 1. MRCP of the abdomen: (A) Axial T2- weighted image showing intra- and extra-hepatic dilatation, (B) Coronal T2-weighted image showing extrahepatic choledochal cyst.

The patient was taken to the operating room for an exploratory laparotomy. A large cystic structure involving the common bile duct was identified and dissected. This large apparent choledochal cyst demonstrated extensive dilation of the common bile duct with typical tapering posteriorly toward the pancreas. The cyst was divided distally and upon attempted drainage, unexpected mucoid "material" (see Figure 2) was removed and submitted for immediate frozen section evaluation in order to provide a provisional histologic diagnosis. The frozen section diagnosis was RMS (see Figure 2). The lesion was transected at the level of the planned anastomosis and an intra-operative cholangiogram was performed. The left hepatic duct was significantly thickened with "debris" within the lumen (see Figure 2). A Roux-en-Y hepaticojejunostomy with lymph node sampling of the porta hepatis was performed. The final pathology diagnosis on examination of all tissue submitted was embryonal RMS, botryoid type. The surgical margins at the left and right hepatic ducts were involved by tumor. There was no lymphovascular invasion and hilar lymph nodes were negative for tumor.

The patient recovered with no complications. Prior to discharge, a computerized tomography (CT) scan of the chest, abdomen and pelvis, and a nuclear bone scan were done. These did not show any evidence of metastatic disease. Bi-
While at home, the patient developed signs and symptoms of cholangitis that included fever, increased abdominal pain, and jaundice. His white blood cell count (WBC) was elevated at $19 \times 10^{3} / \mu 1$ and conjugated bilirubin had increased to $2.7 \mathrm{mg} / \mathrm{dl}$.

lateral bone marrow biopsy and aspirations were performed, and these showed no metastatic tumor. The patient was discharged on post-operative day 14 after the first course of chemotherapy. The patient has completed treatment according to Children's Oncology Group (COG) ARST0531 protocol, regimen A consisting of vincristine, dactinomycin, and cyclophosphamide. In addition, he received 50.4 CGE proton beam radiation therapy to the tumor bed. The end-oftherapy imaging scans did not show any evidence of residual disease, recurrence or metastatic disease.

\section{Discussion}

RMS is the most common soft tissue sarcoma of childhood and occurs in a variety of anatomic locations. ${ }^{[8]}$ RMS of the biliary tree is rare, accounting for $<1 \%$ of all pediatric RMS. In spite of this, RMS is the most common malignant tumor of the biliary tree in childhood, with $75 \%$ of cases reported in children less than 5 years of age and with a male predominance. ${ }^{[2,5]}$

RMS can originate anywhere along the biliary tree, resulting in signs and symptoms of obstructive jaundice. Jaundice is observed in $60 \%-80 \%$ of the cases. ${ }^{[5]}$ Other symptoms include abdominal pain, emesis, fever, acholic stools, and decreased appetite. ${ }^{[9]}$ Similar to the present case, choledochal cyst is the most common clinical and radiologic diagnosis 
prior to surgery, according to a report from the Intergroup Rhabdomyosarcoma Study (IRS). ${ }^{[2]}$ Abdominal US is typically performed as the initial imaging modality for the workup of pediatric patients and often reveals dilated intra and extrahepatic bile ducts with a hyperechoic intraluminal mass within the ductal system. Color Doppler is useful if internal flow is adequately demonstrated within the intraluminal mass, which allows differentiation from etiologies other than a solid lesion. However, the appearance of biliary RMS on CT imaging can be highly variable, presenting as either a homogeneous or heterogeneous, hypoattenuated -or hyperattentuated mass. On MRI T1-weighted images, RMS will present as a $\mathrm{T} 1$ hypointense and $\mathrm{T} 2$ hyperintense mass compared to the liver parenchyma. ${ }^{[10]}$ Post-contrast imaging is usually helpful since the presence of enhancement is a key feature that differentiates RMS from benign entities like sludge or debris. The role of transhepatic cholangiography or endoscopic retrograde cholangiopancreatography (ERCP) in the diagnosis of biliary RMS is still unclear. A recent publication reported on the use of ERCP as a technique for obtaining a diagnostic biopsy in two patients with suspected RMS. The authors suggested that intraluminal biopsy of a biliary tract tumor employing ERCP may minimize the risk of local dissemination; however this remains anecdotal and controversial. ${ }^{[1]}$

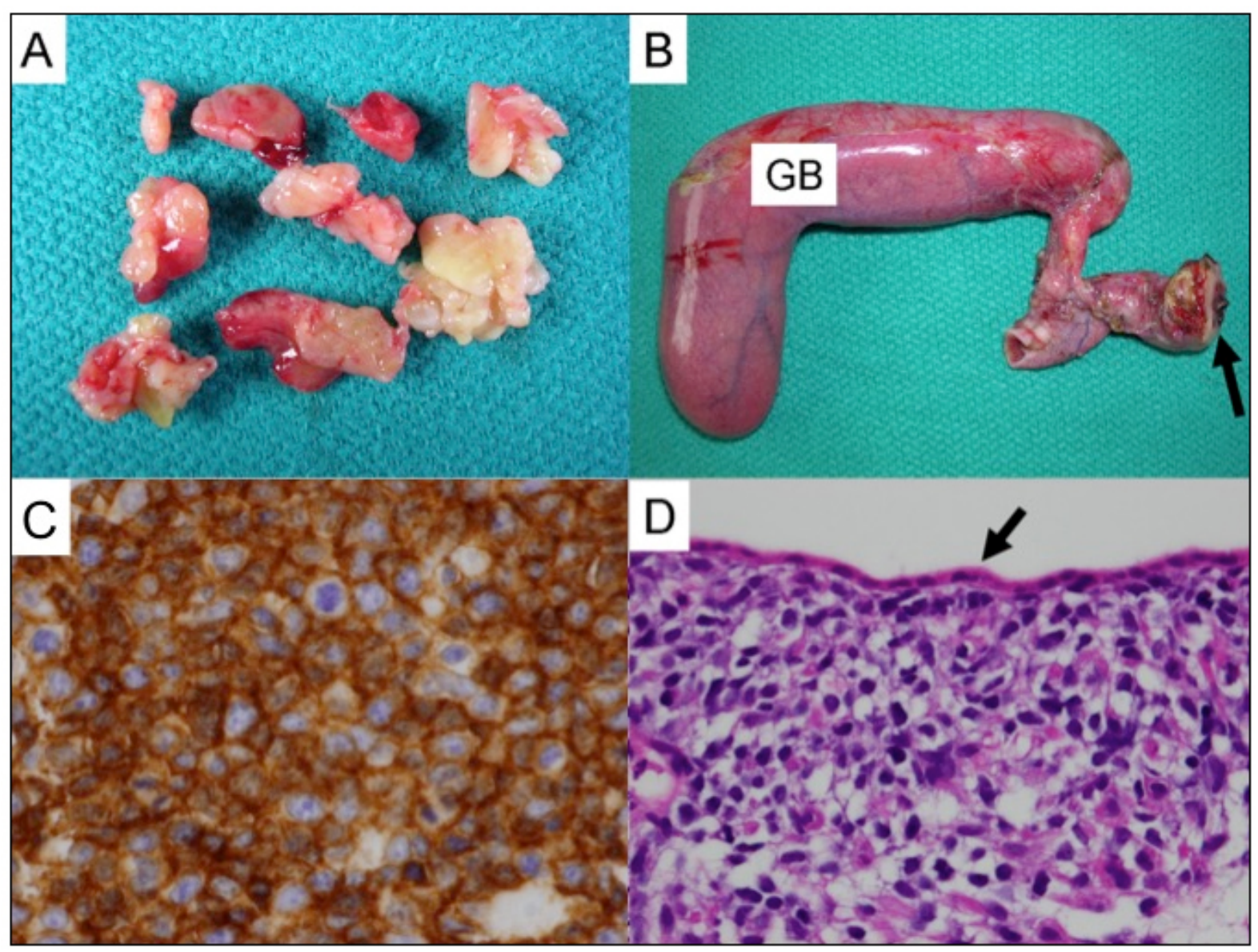

Figure 2. Gross and histopathologic appearance of biliary tract rhabdomyosarcoma

A: Intraluminal soft tissue submitted for frozen section diagnosis; B: Resection of gallbladder (GB) with associated dilated biliary tree mimicking a choledochal cyst. Note the presence of red tan friable tumor at one of the resection margins (arrow); C: Tumor cells with diffuse cytoplasmic immunoreaction with desmin antibody, indicative of rhabdomyoblasts (400×); D: Botryoid rhabdomyosarcoma characterized by an attenuated epithelial lining (arrows) with underlying cambium layer of undifferentiated rhabdomyoblasts and differentiating rhabdomyoblast with dense eosinophil cytoplasm.

Pediatric RMS presents as two distinct histopathologic subtypes, embryonal (ERMS) and alveolar (ARMS). ${ }^{[1]}$ This classification serves as an independent predictor of clinical

outcome and is an important component of risk stratification in RMS. ${ }^{[12]}$ ERMS is the most common subtype of RMS and is generally associated with better outcomes. ${ }^{[1]}$ ERMS 
is further comprised of a range of histologies based on the degree of embryonic rhabdomyogenesis. Botryoid RMS, a type of ERMS, forms grape-like polyploidy masses with their surfaces partially lined by epithelium. ${ }^{[12]}$ Biliary RMS is typically botryoid embryonal RMS type, which is more sensitive to chemotherapy. ${ }^{[2,9]}$

Although previously authors have argued for aggressive surgery in the treatment of biliary RMS, this is not always feasible and can lead to significant morbidity. In a review of the IRS data from 1972-1998, only 6 of 25 patients had gross total resection and only 2 had negative surgical margins on microscopic examination. Despite the presence of positive surgical margins, 13 of 14 patients with gross residual disease after surgery remained free of disease, following oncologic management. Based on these findings, aggressive surgical resection in biliary RMS is discouraged. The primary goals of surgical intervention should be to establish an accurate diagnosis and to determine the local-regional extent of disease. ${ }^{[9]}$ Once believed to be an incurable disease, the outcome for patients with biliary RMS has improved with a multidisciplinary treatment approach, including appropriate surgery, chemotherapy, and radiation. ${ }^{[2]}$ For patients with local-regional disease, as with the present case, the estimated 5 -year overall survival is $78 \%$. ${ }^{\text {[9] }}$

In young children presenting with symptoms of obstructive jaundice or with an initial diagnosis of choledochal cyst, biliary RMS should be in the differential diagnosis. Although rare, it remains the most common malignancy of the biliary tract system in childhood. Treatment is multimodal, and aggressive surgical resection is not warranted and does not improve outcomes.

\section{Conflicts OF InTEREST Disclosure}

The authors declare no conflicts of interest.

\section{REFERENCES}

[1] Hawkins DS, Spunt SL, Skapek SX. Children's Oncology Group's 2013 blueprint for research: Soft tissue sarcomas. Pediatr Blood Cancer. 2012; 60: 1001-8. PMid: 23255356. http://dx.doi.org $/ 10.1002 / \mathrm{pbc} .24435$

[2] Ruymann FB, Raney RB Jr, Crist WM, et al. Rhabdomyosarcoma of the biliary tree in childhood. A report from the Intergroup Rhabdomyosarcoma Study. Cancer. 1985; 56: 57581. http://dx.doi.org/10.1002/1097-0142(19850801)56: 3<575: : AID-CNCR2820560326>3.0.CO;2-9

[3] Kebudi R, Gorgun O, Ayan I, et al. Rhabdomyosarcoma of the biliary tree. Pediatr Int. 2003; 45: 469-71. PMid: 12911487. http: //dx.doi.org/10.1046/j.1442-200X.2003.01763.x

[4] Perera MT, McKiernan PJ, Brundler MA, et al. Embryonal rhabdomyosarcoma of the ampulla of vater in early childhood: report of a case and review of the literature. J Pediatr Surg. 2009; 44: 9-11. PMid: 19231522. http://dx.doi.org/10.1016/j.jpedsurg. 2008.10.113

[5] Ali S, Russo MA, Margraf L. Biliary rhabdomyosarcoma mimicking choledochal cyst. J Gastrointestin Liver Dis. 2009; 18: 95-7. PMid: 19337643.

[6] Tireli GA, Sander S, Dervisoglu S, et al. Embryonal rhabdomyosarcoma of the common bile duct mimicking choledochal cyst. J Hepatobiliary Pancreat Surg. 2005; 12: 263-5. PMid: 15995817. http://dx.doi.org/10.1007/s00534-004-0959-7
[7] Zampieri N, Camoglio F, Corroppolo M, et al. Botryoid rhabdomyosarcoma of the biliary tract in children: a unique case report. Eur J Cancer Care. 2006; 15: 463-6. PMid: 17177904. http://dx.doi.org/10.1111/j.1365-2354.2006.00683.x

[8] Mollen K, Rodeberg DA. Diagnosis and treatment of rhabdomyosarcoma. In: Coran AG (ed) Pediatric Surgery. 7th edn. Philadelphia: Elsevier; 2012. http://dx.doi.org/10.1016/B978-0-323-0 7255-7.00035-0

[9] Spunt SL, Lobe TE, Pappo AS, et al. Aggressive surgery is unwarranted for biliary tract rhabdomyosarcoma. J Pediatr Surg. 2000; 35: 309-16. http://dx.doi.org/10.1016/S0022-3468(00) 9 0030-7

[10] Chung EM, Lattin GE Jr, Cube R, et al. From the archives of the AFIP: Pediatric liver masses: radiologic-pathologic correlation. Part 2. Malignant tumors. Radiographics. 2011; 31: 483-507. PMid: 21415193. http://dx.doi.org/10.1148/rg.312105201

[11] Scottoni F, DeAngelis P, Dall'Oglio L, et al. ERCP with intracholdocal biopsy for the diagnosis of biliary tract rhabdomyosarcoma in children. Pediatr Surg Int. 2013; 29: 659-62. PMid: 23417547. http://dx.doi.org/10.1007/s00383-013-3282-z

[12] Parham DM, Barr FG. Classification of rhabdomyosarcoma and its molecular basis. Adv Anat Pathol. 2013; 20: 387-97. PMid: 24113309. http://dx.doi.org/10.1097/PAP.0b013e3 182a92d0d 